\title{
Shocks and Discontinuities Treatment in Multi-species Flows Using a Riemann Solver.
}

\author{
Hind Benakrach ${ }^{1, *}$, Mohamed Bounouib ${ }^{1}$, Mourad Taha-Janan ${ }^{1}$, and Mohamed Zeriab Essadek ${ }^{1}$ \\ ${ }^{1}$ Laboratory of Applied Mechanics and Technologies (LaMAT), ENSAM, STIS Research Center; Mohammed V University in Rabat, \\ Morocco
}

\begin{abstract}
The diversity of industrial configurations presenting a flow of mixtures of several species calls on researchers to develop a reliable, easily usable tool capable of treating this type of flow in the presence of an interface. The present work contributes to the numerical solution of multi-species equations in the presence of shocks and contact discontinuities. A shock capture-type method based on the separation of roe flux differences is associated with a two-parameter equation of state. The results presented here show that the model has the ability to correctly capture the present shocks and discontinuities and provide an accuracy compatible with the requirements zones of discontinuity existing in the flow.
\end{abstract}

\section{Introduction}

Among the main challenges of numerical simulation is its ability to deal with different flows despite their complicity. Flows containing shocks and contact discontinuities are one of those complex flows that occur in various industrial configurations. It is therefore desirable to be able to predict it with great precision without this being costly.

The simulation of these complex flows needs to consider the different physical phenomena to approach real cases. In the case of a multi-species flow, the complexity of the flow increases, and the number of equations can rapidly weigh down the calculation, which makes the selection of equations a critical step in the simulation.

At the level of formulations, the models implemented are approaching more and more the most realistic models; the high-performance numerical diagrams are then the most requested for the resolution of the equations which describe this type of complex flows $[1,2]$.

For reasons of stability and precision, the finite volume method was applied during the present study. A class of methods based essentially on the resolution of the Riemann problem has been retained.

Roe's method of separation of flow differences has been very successful despite being expensive. It has the advantage of adapting to compressible and incompressible fluids. In addition, it pairs easily with a broad category of equations of state [3].

The association of this method with the gamma model [4] has shown its effectiveness for mono-species flows [5], as well as for multi-species flows described in a curvilinear reference [6].
This gamma model brings its flexibility to simulate the compressible and incompressible fluid by a single formulation.

This present work aims to treat multi-species flows of a non-viscous, compressible or incompressible fluid with a single formulation. Several limiters have been introduced into the digital flux expression to remove fluctuations and bring back the precision and the sharpness near the zones of shocks.

\section{Mathematical formulation}

To describe a two-dimensional flow of a non-viscous fluid, we consider Euler's equations written in a Cartesian coordinate system. The conservative form of these equations can be written as:

$$
\frac{\partial}{\partial t}\left(\begin{array}{c}
\rho \\
\rho v \\
\rho E_{0}
\end{array}\right)+\frac{\partial}{\partial x}\left(\begin{array}{c}
\rho u \\
\rho u^{2}+p \\
\rho u v \\
\left(\rho E_{0}+p\right) u
\end{array}\right)+\frac{\partial}{\partial y}\left(\begin{array}{c}
\rho v \\
\rho u v \\
\rho v^{2}+p \\
\left(\rho E_{0}+p\right) v
\end{array}\right)=0
$$

Where:

- $\quad \rho:$ The density of the assumed fluid;

- $u$ and $v$ : The components of the velocity vector in the considered Cartesian reference;

- $\quad p:$ The static pressure;

- $E_{0}$ : The total mass-energy expressed as:

$$
E_{0}=e+\frac{u^{2}+v^{2}}{2}
$$

${ }^{e}$ represents the internal energy mass of the fluid. 
The equation of state carries out the closing of the system of equation with two parameters $\gamma$ and $p_{\infty}$, and it is written as follows:

$$
\rho e=\frac{p+\gamma p_{\mathrm{se}}}{\gamma-1}
$$

$\gamma$ and $p_{\infty}$ are values determined from empirical correlations for each species or component.

The global system of equations governing the nonviscous multi-species flow is written in the following vector and conservative form:

$$
\frac{\partial q}{\partial t}+\frac{\partial E}{\partial x}+\frac{\partial F}{\partial y}=0
$$

$q$ represents the conservative variables:

$$
q=\left(\begin{array}{c}
\rho \\
\rho u \\
\rho v \\
\rho E_{0} \\
\frac{1}{\gamma-1} \\
\frac{\gamma p_{\infty}}{\gamma-1}
\end{array}\right)
$$

$E$ and $F$ represent the inviscid fluxes:

$E=\left(\begin{array}{c}\rho u \\ \rho u^{2}+p \\ \rho u v \\ \left(\rho E_{0}+p\right) u \\ \frac{u}{\gamma-1} \\ \frac{u \gamma p_{\infty}}{\gamma-1}\end{array}\right), F=\left(\begin{array}{c}\rho v \\ \rho u v \\ \rho v^{2}+p \\ \left(\rho E_{0}+p\right) v \\ \frac{v}{\gamma-1} \\ \frac{v \gamma p_{\infty}}{\gamma-1}\end{array}\right)$

\section{Numerical approximation}

The first numerical method capable of taking into account discontinuities is due to Godunov. Based on an explicit off-center scheme, Godunov [7, 8] suggested solving the Riemann problem at each time step by keeping all the conservative variables constant on each mesh. This technique involves a very complex structure due to the local solution of the Riemann problem. The resolution, therefore, becomes very expensive.

In [4], Roe proposes a modification of the Godunov scheme by determining the solution of the non-linear problem by a linear transformation. The Roe matrix, therefore, replaces the Jacobian matrix $(\tilde{E}(q), F(q))$, the linear system is then written as follows:

$\frac{\partial q}{\partial t}+E(q) \frac{\partial q}{\partial x}+F(q) \frac{\partial q}{\partial y}-0$

The expression of the numerical flux of convective vectors is written in the form:

$$
\left\{\begin{array}{l}
E_{i+1 / 2}^{*}=\frac{1}{2}\left(\bar{E}_{i}+\bar{E}_{i+1}\right)+D_{i+\frac{1}{z}} \\
F_{j+1 / 2}^{*}=\frac{1}{2}\left(\bar{F}_{j}+\bar{F}_{j+1}\right)+D_{j+\frac{1}{2}}
\end{array}\right.
$$

$D_{i+\frac{1}{2}}$ and $D_{j+\frac{1}{2}}$ are the terms which determine the type and the order of precision of the discretization [9]. These terms are given by:

$$
\begin{aligned}
& D_{i+\frac{1}{2}}=-\frac{1}{2}\left|\widetilde{\mathrm{A}}_{i+\frac{1}{2}}\right|\left(q_{i+1}-q_{i}\right) \\
& +\frac{1}{4}\left\{(1-\kappa) \Psi_{i-\frac{1}{2}}^{+} \widetilde{\mathrm{A}}_{i-\frac{1}{2}}^{+}\left(q_{i}-q_{i-1}\right)\right. \\
& +(1+\kappa) \Psi_{i+\frac{1}{2}}^{-} \widetilde{\mathrm{A}}_{i+\frac{1}{2}}^{+}\left(q_{i+1}-q_{i}\right) \\
& -(1-\kappa) \Psi_{i-\frac{1}{2}}^{+} \widetilde{\mathrm{A}}_{i+\frac{1}{2}}^{-}\left(q_{i+1}-q_{i}\right) \\
& \left.-(1-\kappa) \Psi_{i+\frac{3}{2}}^{-} \widetilde{\mathrm{A}}_{i+\frac{3}{2}}^{-}\left(q_{i+2}-q_{i+1}\right)\right\} \\
& D_{j+\frac{1}{2}}=-\frac{1}{2}\left|\widetilde{\mathrm{B}}_{j+\frac{1}{2}}\right|\left(q_{j+1}-q_{j}\right) \\
& +\frac{1}{4}\left\{(1-\kappa) \Psi_{j-\frac{1}{2}}^{+} \widetilde{\mathrm{B}}_{j-\frac{1}{2}}^{+}\left(q_{j}-q_{j-1}\right)\right. \\
& +(1+\kappa) \Psi_{j+\frac{1}{2}}^{-} \widetilde{\mathrm{B}}_{j+\frac{1}{2}}^{+}\left(q_{j+1}-q_{j}\right) \\
& -(1-\kappa) \Psi_{j-\frac{1}{2}}^{+} \widetilde{\mathrm{B}}_{j+\frac{1}{2}}^{-}\left(q_{j+1}-q_{j}\right) \\
& \left.-(1-\kappa) \Psi_{j+\frac{3}{2}}^{-} \widetilde{\mathrm{B}}_{j+\frac{3}{2}}^{-}\left(q_{j+2}-q_{j+1}\right)\right\}
\end{aligned}
$$

$\widetilde{\mathrm{A}}_{i+\frac{1}{2}}$ and $\widetilde{\mathrm{B}}_{j+\frac{1}{2}}$ are called Roe's matrices.

$\Psi$ is given by the expression for the limiter in the presence of shocks or contact discontinuities. When the parameter $\kappa$ makes it possible to adjust the order of precision of the discretization.

Three types of limiters will intervene along with this work, namely:

- Van Leer limiter (1974) [10]:

$$
\Psi(r)=\frac{r+|r|}{1+r}
$$

- $\quad$ Van Albada limiter (1997) [11]:

$$
\Psi(r)=\frac{r^{2}+r}{1+r^{2}}
$$

- $\quad$ "Superbee" by Roe limiter (1985) [12] :

$$
\Psi(r)=\max [0, \min (2 r, 1), \min (r, 2)]
$$

$r$ represents the ratio of successive gradients on the solution mesh.

\section{Numerical tests}

The previous section shows that the proposed model contains several resolution options depending on the required precision order. These options change depending on the chosen limiter as well as the order of spatial discretization precision used. A series of simulations were performed to assess the solver's ability to capture shocks and discontinuities correctly.

\subsection{Riemann gas-liquid problem}

Cocchi and Saurel [13] have treated an unsteady interface case. The initial state is as follows: 
- For gas, on the left: $0 \leq x<0.5$

$\left(\rho, u, v, p, \gamma, p_{\infty}\right)_{L}=(1.241,0,0,2.753,1.4,0)$

- For liquid, on the right: $0.5 \leq x<1$

$\left(\rho, u, v, p, \gamma, p_{\infty}\right)_{R}=\left(0.991,0,0,3.059 \times 10^{-4}, 5.4,1.505\right)$

Knowing that the left portion contains the gas and the right side contains the liquid, the shock wave, and the discontinuity wave move from left to right, generating a refractive wave in the gas.

The computation of the variables was carried out on a mesh comprising [300 x 2] points with a time step of $2.10^{-4}$.

These first results (Fig. 1) linked to the choice of the limiter and the order illustrate the excess diffusion introduced with a $1^{\text {st }}$ order decentring by bringing a smooth aspect to the discontinuity. For $3^{\text {rd }}$ order, an appearance of oscillations close to the discontinuity compared to the $2^{\text {nd }}$ order.

Regarding the limiters, note that the Van Leer limiter and the Van Albada limiter, associated with the $2^{\text {nd }}$ order, provided the right combination for better shock capture and discontinuity.
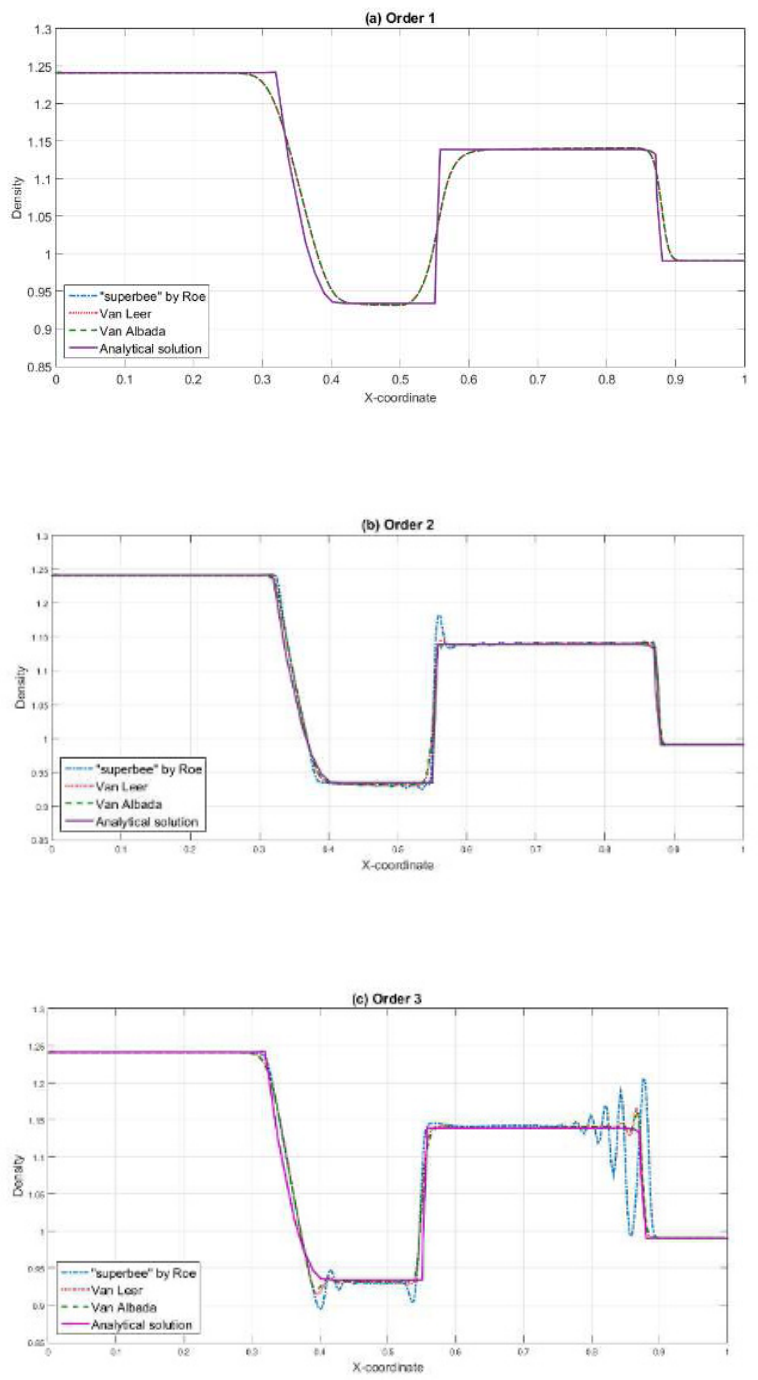

Fig. 1. Comparison of analytical and numerical results calculated with (a) $1^{\text {st }}$ Order, (b) $2^{\text {nd }}$ Order, and (c) $3^{\text {rd }}$ Order, using different limiters: "Superbee" by Roe, Van Leer, and Van Albada.
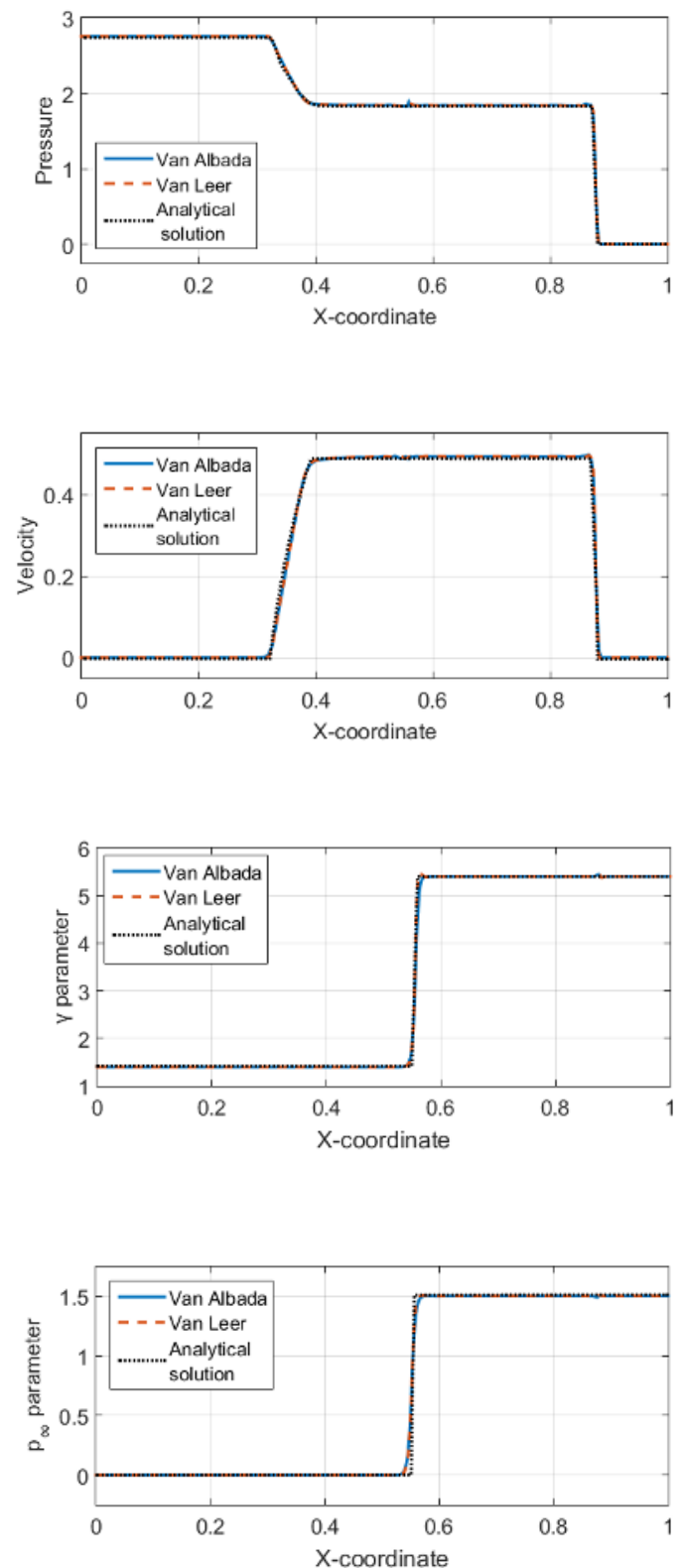

Fig. 2. Comparison of analytical and numerical results using Van Albada and Van Leer limiters for pressure, velocity-u,

$p_{\infty}$, and $\gamma$ parameters.

From Fig.2, the distribution highlights the position of the contact discontinuity, which proves that the two limiters can deal with interface problems between two different phases, namely gas and liquid.

\subsection{D interface problem}

For a two-dimensional test, consider a circle of radius 0.16 placed at $x_{0}=y_{0}=0.25$ of a square of [0:1]x [ $\left.0: 1\right]$.

- On the outside of the disc, the fluid is characterized by:

$$
\left(\rho, u, v, p, \gamma, p_{\infty}\right)_{r \measuredangle r_{0}}=(1,1,1,1,1.4,0)
$$


- While inside, we have:

$\left(\rho, u, v, p, \gamma, p_{\infty}\right)_{r r_{0}}=(0.125,1,1,1,4,1)$

In a regular mesh characterized by $\Delta x=\Delta y=0.01$. The Van Leer limiter was used.

Figure 3 represents the results obtained compared to the results obtained by shyue [4].

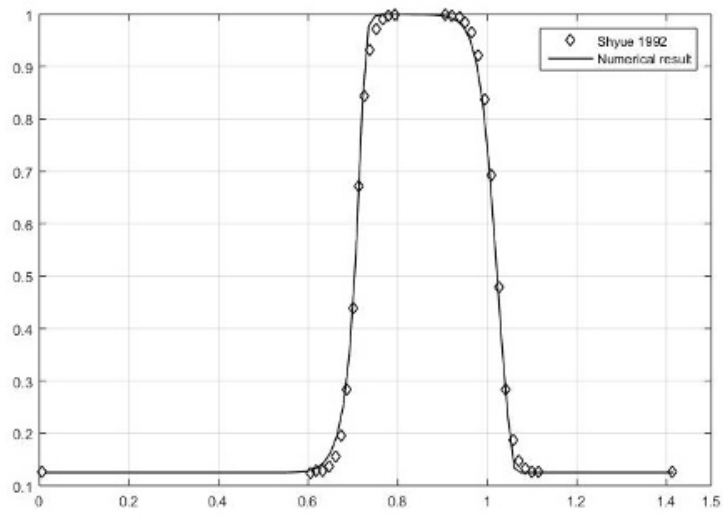

Fig. 3. Density comparison obtained at $t=0.36$ to that obtained by Shyue[4].

Figure 3 shows the density results obtained at $t=0.36$ along the diagonal $(\mathrm{x}=\mathrm{y})$. This severe test case passes without problem with results comparable to those presented in the literature.

\section{Conclusion}

The use of the Roe flux difference separation technique was the subject of this study. This approach is effective in treating two-dimensional multi-species flows for nonviscous, compressible, and incompressible fluids. The different test cases treated in this work have shown the method's ability to capture shocks and contact discontinuities with high accuracy.

\section{References}

1. B.VAN LEER, Towards the ultimate conservative difference scheme. V. A second-order sequel to Godunov's method. Journal of computational Physics, vol. 32, no 1, p. 101-136 (1979)

2. S. YAMAMOTO DAIGUJI, H. Higher-orderaccurate upwind schemes for solving the compressible Euler and Navier-Stokes equations. Computers \& Fluids, vol. 22, no 2-3, p. 259-270 (1993)

3. P.L. ROE, The use of the Riemann problem in finite difference schemes. In: Seventh International Conference on Numerical Methods in Fluid Dynamics. Springer, Berlin, Heidelberg, 1989. p. 354-359.

4. K. SHYUE, An efficient shock-capturing algorithm for compressible multicomponent problems. Journal of
Computational Physics, vol. 142, no 1, p. 208-242 (1998)

5. P.L. ROE, Approximate Riemann solvers, parameter vectors, and difference schemes. Journal of computational physics, vol. 43, no 2, p. 357-372 (1981)

6. H.BENAKRACH, M. TAHA-JANAN, M.Z. ESSADEK, simulation of compressible and incompressible flows in the presence of shocks. In: MATEC Web of Conferences. EDP Sciences. p. 07018 (2019)

7. M. Taha Janan and A. El Marjani, "A flow solver for the Euler and Navier-Stokes equations for multi-phase flows with a stiffened gas equation of state," International Journal of Numerical Methods for Heat \& Fluid Flow, vol. 17, no. 8, pp. 823-835( 2007)

8. S.K. Godunov,A difference method for the calculation of shock waves. Amer. Math, Soc. Transl, 16(2):389390,30 (1960)

9. R. Baraille, J.M. Greenberg, A.Y. Leroux, A. Noussair, Analysis and approximation of conservation laws with source terms. J. Num. Anal., 35(N5): 19802007. 49 (1997)

10. B. VAN LEER, Towards the ultimate conservative difference scheme. II. Monotonicity and conservation combined in a second-order scheme. Journal of computational physics, vol. 14, no 4, p. 361-370 (1974)

11. G. D VAN ALBADA, B. VAN LEER, W. ROBERTS, A comparative study of computational methods in cosmic gas dynamics. In : Upwind and highresolution schemes. Springer, Berlin, Heidelberg, p. 95103 (1997)

12. P.L. ROE, Modelling of Discontinuous Flows. Lectures in Applied Mathematics, vol. 22 (1985)

13. J-P. COCCHI, R. SAUREL, A Riemann problem based method for the resolution of compressible multimaterial flows. Journal of Computational Physics, vol. 137 , no 2 , p. 265-298 (1997) 